\title{
Laser Emission from Electrospun Polymer Nanofibers**
}

\author{
Andrea Camposeo, Francesca Di Benedetto, Ripalta Stabile, Antonio A. R. Neves, \\ Roberto Cingolani, and Dario Pisignano*
}

One-dimensional (1D) photonic nanostructures ${ }^{[1]}$ are attracting a growing interest because they can be employed in components of sub-wavelength size for light generation, confinement, guiding, and detection. In particular, organic ${ }^{[2]}$ and inorganic $^{[3]}$ nanowires are $1 \mathrm{D}$ elements capable of realizing both active and passive photonic circuits. The properties of synthesized nanowires have been widely investigated, which has led to the demonstration, for instance, of 1D elongated nanoparticles suitable for the light amplification through stimulated emission. ${ }^{[4]}$ Upon controlled assembly ${ }^{[3 \mathrm{~d}, \mathrm{e}]}$ and manipulation, ${ }^{[5]}$ nanowires have been utilized complementary to photonic band-gap structures and plasmonic-based devices in nanophotonics, ${ }^{[6]}$ relying instead on optical elements directly fabricated on chip.

Among other nanostructures, polymer nanofibers ${ }^{[7]}$ are novel, cheap, and flexible building blocks potentially employable in functional, optical, and electronic nanoscale circuits. They can be fabricated by different approaches, ${ }^{[8]}$ although electrospinning (ES), based on the stretching of a polymer solution under electrostatic forces, represents a practically unique technology that combines low cost and high throughput. Moreover, the addition of active components (i.e., nanoparticles or molecular species) to the ES polymer solution allows one to obtain composite nanofibers with specific functionalities. ${ }^{[7,9,10]}$ In particular, recently achieved light-emitting electrospun nanofibers ${ }^{[9-12]}$ are currently under study for their waveguiding properties, ${ }^{[10]}$ and for their photo- ${ }^{[11,12]}$ and electroluminescence. ${ }^{[9]}$ These nanofibers can be assembled in ordered structures and arrays, ${ }^{[13,14]}$ and they can potentially be used to realize fully plastic, nano-/ microscale optical devices and circuits.

Integrated nanophotonic systems would especially benefit from the availability of nanoscale laser sources, which are

[*] Dr. D. Pisignano, Dr. A. Camposeo, Dr. F. Di Benedetto,

Dr. R. Stabile, Dr. A. A. R. Neves, Prof. R. Cingolani

NNL, National Nanotechnology Laboratory of

Istituto Nazionale di Fisica della

Materia-Consiglio Nazionale delle Ricerche

Università del Salento

via Arnesano, 73100 Lecce (Italy)

E-mail: andrea.camposeo@unile.it

Dr. D. Pisignano

Scuola Superiore ISUFI Università del Salento

via Arnesano, 73100 Lecce (Italy)

[**] We gratefully acknowledge the financial support from the FIRB Contract RBIP06SH3W.

DOI: $10.1002 /$ smll.200801165 characterized by high monocromaticity, directionality, and coherency, and are crucial for optical information processing. ${ }^{[15]}$ Achieving miniaturized light-emitting sources is also gathering interest for integration in sensors and labon-chips. ${ }^{[16]}$ So far, the achievement of organic, 1D nanoscale lasers is still limited to poly(9,9-dioctyfluorene), parasexiphenyl, and 2,4,5-triphenylimidazole nanowires. ${ }^{[17]}$ These nanostructures are synthesized by low volume solution processing under inert atmosphere, followed by melt-assisted template pore wetting, ${ }^{[17 a]}$ or by vacuum sublimation growth at high temperature with typical rates of fraction of $\AA \mathrm{s}^{-1}$. $\left.17 \mathrm{~b}\right]$ For these reasons, cheap, easy, and high-throughput fabrication routes allowing one to obtain $1 \mathrm{D}$ organic lasing media deserve to be explored in depth. The one-step, massive realization of flexible gain nanofibers by ES in air may enable the large-scale production of organic lasing building blocks and the full integration of coherent polymer sources in optical microsystems and disposable diagnostics. In this Communication, we report on lasing action by composite electrospun polymer nanofibers emitting in the visible and near-infrared (NIR) spectral region. The remarkable potential of electrospun, flexible gain nanofibers as active waveguides and lasers are presented. As a prototype system, we demonstrate singlemode optically pumped lasing sources based on individual electrospun nanofibers emitting at $585 \mathrm{~nm}$ with linewidth of $0.3 \mathrm{~nm}$ and excitation threshold fluence as low as $60 \mu \mathrm{J} \mathrm{cm}{ }^{-2}$.

The morphology of our fibers, imaged by scanning electron microscopy (SEM), is shown in Figure 1. Nanofibers with diameter $\left(d_{\mathrm{f}}\right)$ ranging from $50 \mathrm{~nm}$ to a few hundreds of nm are produced, depending on the applied voltage and the solution feeding flow (Figure 1c and d). By increasing the applied electric field, $E$, we obtain smaller diameters (average values of $d_{\mathrm{f}}=190$ and $500 \mathrm{~nm}$ at an applied voltage of 27 and $15 \mathrm{kV}$, respectively) and narrower size distributions (standard deviation $=70$ and $100 \mathrm{~nm}$, respectively). The solution feeding flow, $\phi$, can also be used to control $d_{\mathrm{f}}$. For instance, at a fixed applied voltage of $24 \mathrm{kV}$, the average fiber diameter changes from 190 to $220 \mathrm{~nm}$ upon increasing the injection rate from 1 to $10 \mu \mathrm{L} \mathrm{min}{ }^{-1}$. These findings are consistent with theoretical models for thinning viscoelastic jets of Newtonian solutions accelerated by electric fields, ${ }^{[18]}$ which predict $d_{\mathrm{f}}$ scaling as $(\phi / E)^{2 / 3}$. The single fiber surface is found to exhibit a rootmean-square roughness of about $6 \mathrm{~nm}(\lambda / 10)$ using atomic force microscopy (AFM).

The absorption and photoluminescence (PL) spectra of Rhodamine 6G (R6G)-doped polymer nanofibers are shown in Figure $2 \mathrm{a}$ and $\mathrm{b}$, respectively. The absorption shows a peak at $530 \mathrm{~nm}$, which is a signature of embedded gain dye 

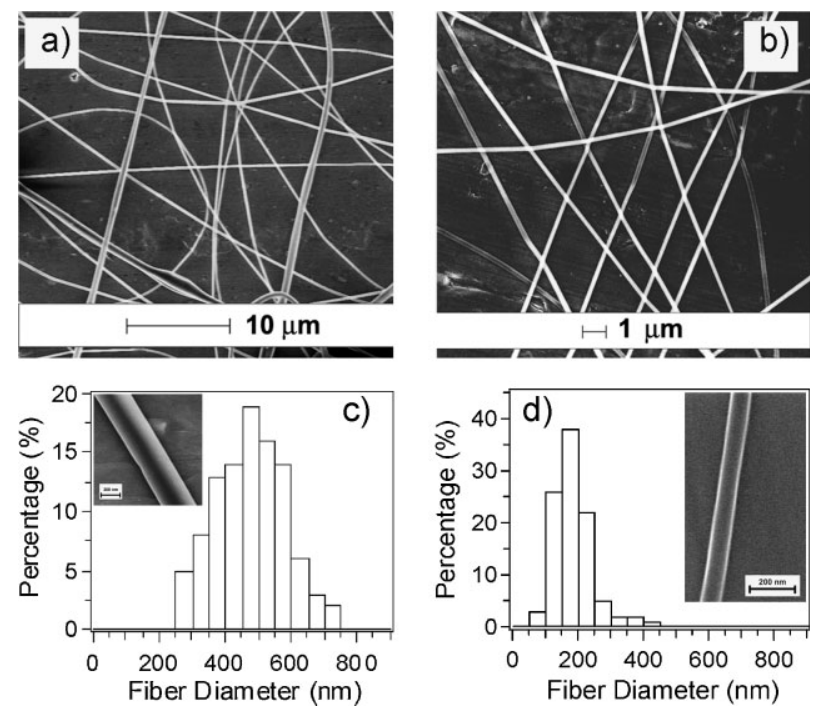

Figure 1. SEM fiber micrographs $(a, b)$ and corresponding diameter distributions (c, d) for mats spun with an applied voltage of 15 and $27 \mathrm{kV}$, respectively. Insets of (c) and (d): SEM images of single nanofibers. Scale bars are 300 and $200 \mathrm{~nm}$, respectively. ES parameters: needle-collector distance $=12 \mathrm{~cm}, \phi=9 \mu \mathrm{L} \mathrm{\textrm {min } ^ { - 1 }}$.
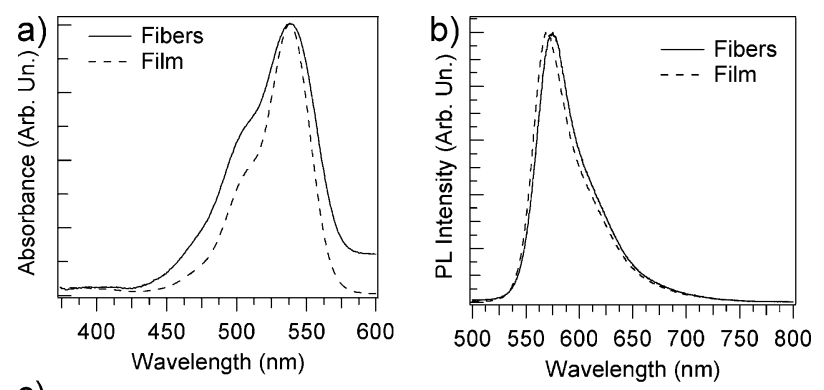

c)



Figure 2. Absorption (a) and PL (b) spectra of fiber mats (continuous lines) incorporating R6G as gain molecule, and of the corresponding reference films (dashed lines). c) FTIR transmission spectra of R6G polymer nanofibers (continuous line) and of a corresponding reference film (dashed line). Spectra are normalized to the intensity of the $1723 \mathrm{~cm}^{-1}$ peak. Inset: zoom-in of the transmission spectra in the range $2800-2960 \mathrm{~cm}^{-1}$. The two vertical lines indicate the position of the peaks at $2843 \mathrm{~cm}^{-1}$ (fiber) and at $2847 \mathrm{~cm}^{-1}$ (film), corresponding to the symmetric stretching mode of $\mathrm{CH}_{2}$.

molecules. The comparison with a reference film evidences a broad spectral component, related to scattered incident light, in the fiber absorption at long wavelengths. Upon continuous wave (CW) excitation of the nanofibers, the PL peak is detected at $570 \mathrm{~nm}$, with characteristic R6G vibronic replicas at $610 \mathrm{~nm}$ and $690 \mathrm{~nm}$. Interestingly, the PL spectrum is red-shifted by about $5 \mathrm{~nm}$ with respect to that of the reference film. Since the local microenvironment surrounding gain compounds can influence the emission wavelength, causing blue- or red-shifts depending on the chemico-physical features (density, refractive index, polarity) of the host material, ${ }^{[19]}$ this finding suggests that the ES process may modify the molecular arrangement in the thermoplastic matrix.

This issue is investigated by Fourier transform infrared (FTIR) spectroscopy (Figure 2c), which evidences softened vibrational modes of host poly(methyl methacrylate) (PMMA) in nanofibers with respect to the bulk film. In the fibers, the $\mathrm{CH}_{2}$ antisymmetric and symmetric stretching modes near 2900 and $2840 \mathrm{~cm}^{-1}$, respectively (inset of Figure 2c), and the $\mathrm{CH}_{2}$ bending modes at $1500-1300 \mathrm{~cm}^{-1}$, which are sensitive to alkyl chain conformation, ${ }^{[19,20]}$ show a shift (by $1-4 \mathrm{~cm}^{-1}$ ) toward lower wavenumbers. These results indicate that the polymer fibers take on a specific conformation with a more ordered organization of lateral chains, as induced by the electrostatic field. ${ }^{[21]}$ In addition, the nanofiber spectra present a large broad band above $1800 \mathrm{~cm}^{-1}$ due to the superposition of modes from $\mathrm{N}-\mathrm{H}$ stretching and $\mathrm{C}-\mathrm{H}$ stretching of the $\mathrm{R} 6 \mathrm{G}$ xanthene ring and phenyl, ethyl, and methyl external groups. $\mathrm{N}-\mathrm{H}$ stretching modes of $\mathrm{R} 6 \mathrm{G}$ are indicative of the formation of hydrogen bonds in the solid state, ${ }^{[22]}$ and suggest an enhanced interaction (with weak bond formation) of the gain compound with the polymer. Such guest-polymer bonds, together with the matrix polarity affected by the matrix arrangement, likely result in the observed red-shift of the nanofiber emission.

Importantly, gain molecules can be embedded in nanofibers without any degradation of the emissive properties, and with remarkable waveguiding properties. We fabricated fibers using many different chromophores that emit light in the VisNIR (Figure 3). The fibers are easily tunable and exhibit a bright and uniform PL, which demonstrates the homogeneous incorporation of the active molecules. These materials can be straightforwardly exploited as nanoscale, incoherent light sources or fluorescent probes. Moreover, by fluorescence microscopy we note that the fiber tips appear brighter than the body, which is a clear evidence of light waveguiding due to the higher refractive index of the polymer $(n=1.49 \text { at } 600 \mathrm{~nm})^{[23]}$ compared to the surrounding air medium (Figure 4). ${ }^{[24]}$ Waveguiding occurs also in bent fibers, with no appreciable increase of the scattered light in the bending region, and we even observed on-axis optical coupling between different fibers (Figure 4a and b). To quantitatively analyze the guiding performances, we measured the propagation losses of radiation by imaging the scattered photons from the surface of single fibers. Assuming uniform and randomly distributed scattering centers throughout the fiber, such scattered light is representative of the attenuation of the optical mode propagating into the waveguide. The losses are determined by measuring the intensity of the light scattered from the fiber surface as a function of the distance, $d$, from the excitation region (Figure 4c). Upon increasing $d$, the PL intensity decreases as $\approx \exp (-\alpha d)$, with a loss coefficient, $\alpha$, in the range $200-1200 \mathrm{~cm}^{-1}$. The exponential decay of light along the fiber axis indicates the predominance of losses due to absorption in 


\section{communications}
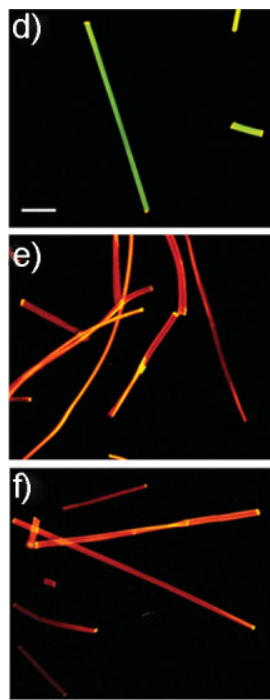

g)
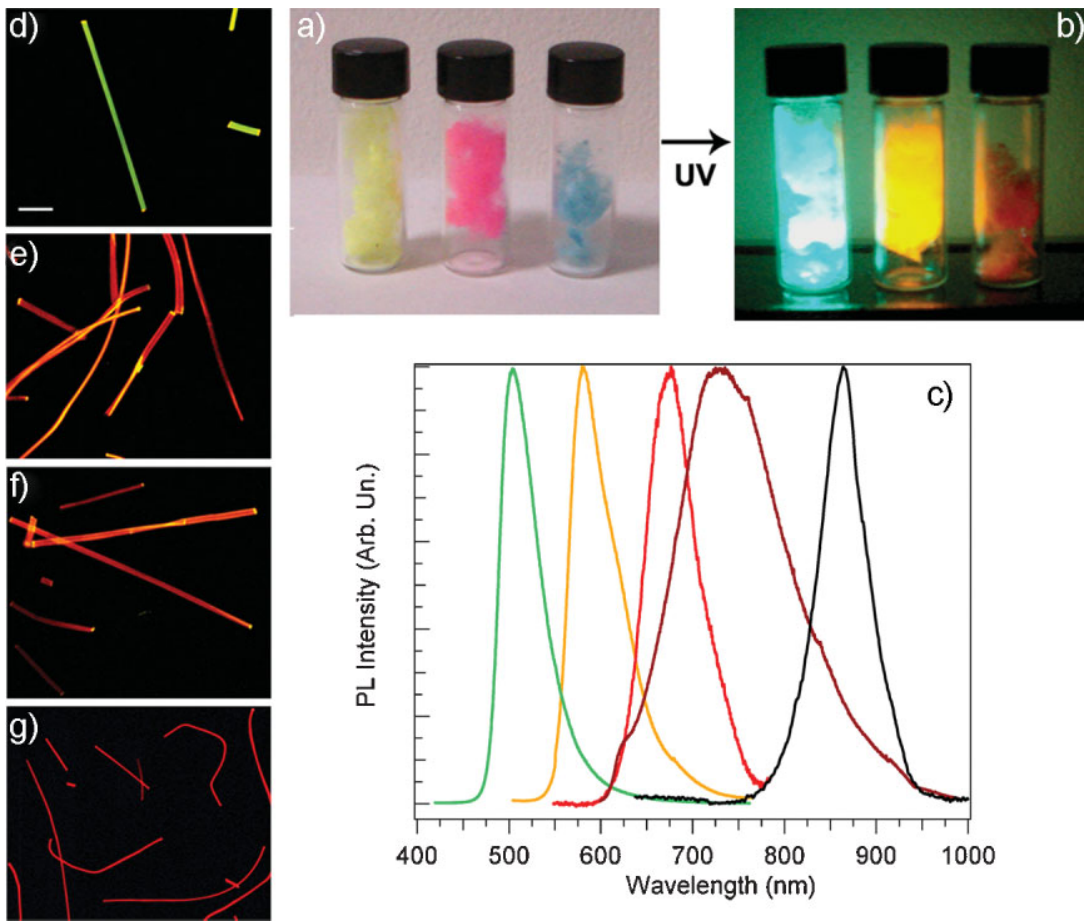

Figure 3. Photographs of gain nanofibers under white (a) and UV (b) light. From left to right, the fibers embed Coumarin 334, R6G, and Nile Blue A Perchlorate. (c) Nanofiber PL spectra under excitation with laser light $(3.05 \mathrm{eV})$. From left to right, spectra from fibers with Coumarin 334, R6G, Nile Blue A Perchlorate, 2-(8-(4-p-Dimetyhlaminophenyl)-2,4-neopentylene1,3,5,7-octatetraenyl)-3-methylbenzothiazolium Perchlorate, and 5,5'-dichloro-11diphenylamino-3,3'-diethyl-10,12-ethylenethiatricarbocyanine Perchlorate. (d-g) Fluorescence images of fibers with Coumarin 334 (d), R6G (e, f), and NileBlue (g). Scale bar: $10 \mu \mathrm{m}$.

in experiments carried out on nanofiber ensembles is due to the fiber size dispersion. In particular, we note that the observed sharp emission peaks cannot be attributed to random lasing effects since the number and spectral positions of the lasing modes remain constant over time and upon an increase in pump fluence. Instead, the spectra of single nanofibers clearly show the spectral features of a Fabry-Pérot cavity, namely equally spaced modes and increased intensity of a particular mode above threshold, such as the mode at $585 \mathrm{~nm}$ with FWHM $0.3 \mathrm{~nm}$ in Figure $5 \mathrm{~d}$. For a cylindrical cavity of length $l$ and central wavelength $\lambda_{\mathrm{c}}$, the mode spacing, $\Delta \lambda_{\mathrm{c}}$, is given by

$$
\Delta \lambda_{\mathrm{c}}=\left(\lambda_{\mathrm{c}}^{2} / 2 l\right)\left(n-\left.\lambda_{\mathrm{c}} \frac{d n}{d \lambda}\right|_{\lambda_{\mathrm{c}}}\right)^{-1}
$$

where $\mathrm{d} n / \mathrm{d} \lambda$ is the refractive index dispersion. By the measured mode spacing $(1.3 \mathrm{~nm})$, we calculate a cavity length of $87 \mu \mathrm{m}$. This is in excellent agreement with the measured fiber length, which confirms that the cavity optical feedback results in the observed lasing of the nanofibers.

In summary, we demonstrate flexible lasers by electrospun gain nanofibers. The

the organics. This is confirmed by the red-shift of the guided PL emission at the fiber tip with respect to light emitted directly from the excited region, because self-absorption is more relevant for emitted photons with higher energy. The measured loss values, comparable to those reported for conjugated polymer nanowires, ${ }^{[25]}$ can be decreased down to fractions of $\mathrm{cm}^{-1}$ by doping active polymer waveguides with donor/acceptor pair materials. ${ }^{[26,27]}$

Finally, the fibers were tested for laser emission. Because of the high confinement of the emitted light, each fiber constitutes a Fabry-Pérot cavity for spectral selection and light amplification. In fact, while the spectra of reference films under pulsed optical excitation peaked at $600 \mathrm{~nm}$ with a full width at half maximum (FWHM) of $20 \mathrm{~nm}$, which is typical of amplified spontaneous emission (ASE) from the gain molecule, ASE from the fibers peaked at $580 \mathrm{~nm}$ with $\mathrm{FWHM}=8 \mathrm{~nm}$ (Figure 5a). This difference is related to the different geometry of the waveguide assisted ASE, which directly affects the spectral properties of the resulting optical amplification. ${ }^{[28]}$ More importantly, for pumping fluences larger than $60 \mu \mathrm{J} \mathrm{cm}^{-2}$, the fiber spectra exhibit sharp emission peaks in the gain region, with a FWHM below $0.5 \mathrm{~nm}$ (Figure $5 b$ ). The intensity of the narrow peaks grows linearly with the pump fluence above threshold (Figure 5c), characteristic of laser emission. This allows us to demonstrate for the first time lasing action from electrospun polymer nanofibers. The presence of a large number of lasing modes fibers show bright luminescence, effective optical waveguiding, and laser emission with a linewidth of $0.3 \mathrm{~nm}$, a threshold

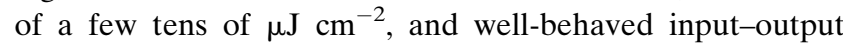
characteristics. The lasing performances, linewidth, and threshold fluence are comparable to state-of-the-art results of macroscopic polymer lasers fabricated by soft lithography and imprinting, ${ }^{[29]}$ but with more potential for integration as a coherent light source in biosensors and microsystems. This is especially interesting in view of embedding excitation sources for chromophores and labeled antibodies flowing within labon-chip microfluidic devices, which to date have been necessarily connected to external lasers or lamps for fluorescence excitation. Furthermore, nanofibers can be assembled in ordered structures, ${ }^{[13,14]}$ allowing the realization of photonic circuits and logic arrays based on coherent, active components. These findings open, therefore, the possibility of high throughput and cost-effective production of fully organic nanoscale photonic systems based on coherent emitters.

\section{Experimental Section}

Nanofibers were fabricated by an ES set-up composed by a syringe, a spinneret (a 18-23 gauge stainless steel needle), a high voltage power supply (10-30 kV), and a grounded aluminum foil used as collector $\left(10 \times 10 \mathrm{~cm}^{2}\right)$. The set-up was mounted on an isolating stand at a variable distance from the needle $(10-30 \mathrm{~cm})$. Gain molecules (0.2-0.5 wt\%) were dissolved in a $1-3 \times 10^{-3} \mathrm{M}$ 

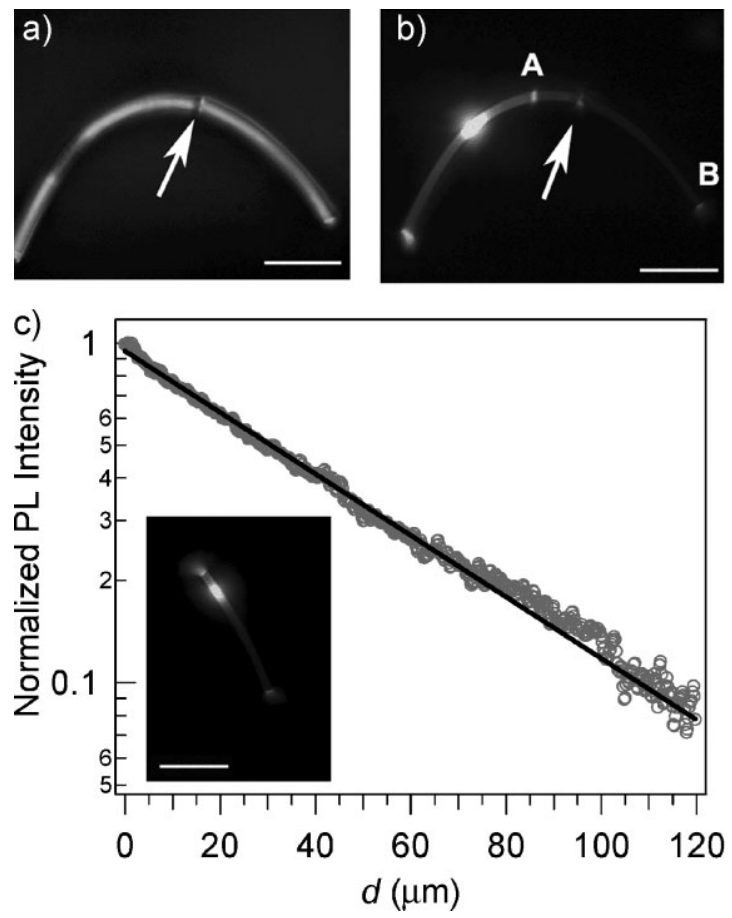

Figure 4. Bright field (a) and fluorescence (b) micrograph of a composite fiber. Scalebar $=10 \mu \mathrm{m}$. The PL is excited by a tightly focused laser (yellow spot in b) and part of the emitted light is coupled in the fiber and observed at the fiber tips (bright spots in b). The spot labeled as $A$ (closest to the excitation spot) is due to scattering from surface defects. The arrow indicates the point of optical coupling between two fibers, evidencing the gap between the two fibers. The spot $B$ is due to light emitted in the first fiber, and exiting from the edge of the second (unexcited) fiber. c) Scattered PL intensity as a function of the distance (d) from the excitation spot. The continuous line is a fit to the data by an exponential decay. Inset: typical fluorescence image of a fiber under loss analysis. Scale bar $=10 \mu \mathrm{m}$.

chloroform solution of PMMA, and the resulting mixture was injected into the syringe by a pump at variable rate $(0.1-10 \mu \mathrm{L}$ $\min ^{-1}$ ). ES was performed at room temperature with about $50 \%$ air humidity, and nonwoven mats of dry nanofibers with typical lengths from 10 to $100 \mu \mathrm{m}$ on quartz substrates were collected. Reference films were spin-cast $(4000 \mathrm{rpm})$ from the same solution. Fiber morphology was investigated by SEM and AFM. SEM analysis was performed by exploiting a Raith 150 electron beam system operating with an acceleration voltage in the range 5-10 kV and an aperture size of $30 \mu \mathrm{m}$. AFM tapping mode measurements were performed by using a Nanoscope Illa controller with a Multimode head (Veeco). Absorption spectra of the nanofibers were collected by a spectrophotometer (Lambda 950, Perkin Elmer) and FTIR spectroscopy measurements were performed by a Spectrum 100 (Perkin Elmer) spectrometer equipped with an attenuated total reflectance accessory, with a mounted Zn-Se crystal for coupling. Fluorescence images were acquired by a stereomicroscope (Leica) using a $\mathrm{Hg}$ lamp as excitation source and a color charge coupled device (CCD) as detector. Waveguiding measurements were carried out by an up-right microscope (Olympus) equipped with a $100 \times$ objective (N.A. 0.9) and a CCD camera. The excitation laser beam was coupled into the microscope objective through a dichroic mirror. The CW PL was measured upon excitation with a CW diode laser $(405 \mathrm{~nm})$ and collection of the emission spectra by a)

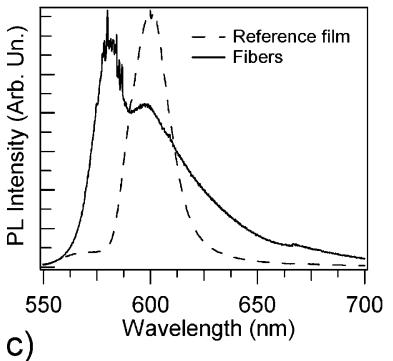

b)
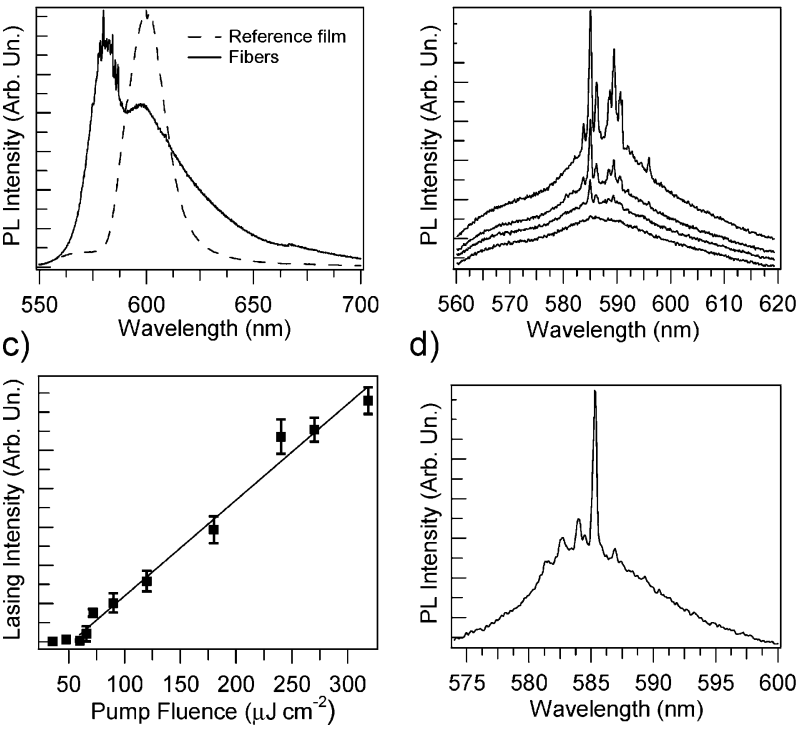

Figure 5. a) ASE spectra of nanofibers (continuous line) and reference film (dashed line). b) Fiber emission spectra for different incident pump fluences. From bottom to top: 50, 65, 70, and $100 \mu \mathrm{Jcm}^{-2}$. c) Laser emission intensity versus excitation fluence. The continuous line is a linear fit to the data in the range $60-350 \mu \mathrm{J} \mathrm{cm}^{-2}$. d) Emission spectrum of a single R6G fiber at a pump fluence of $150 \mu \mathrm{J} \mathrm{cm}{ }^{-2}$.

a fiber-coupled monochromator (iHR320, Jobin Yvon) and a CCD camera. For lasing measurements, the fiber mats were excited by the second harmonic of a Nd:YAG microlaser $(532 \mathrm{~nm}$, pulse duration $0.6 \mathrm{~ns}$, repetition rate $100 \mathrm{~Hz}$ ), whose fluence was attenuated by neutral density filters and measured by a calibrated energy meter (Coherent). The excitation beam was focused on the mats by a cylindrical lens and shaped by a mechanical filter into a rectangular spot $\left(0.30 \times 0.05 \mathrm{~cm}^{2}\right)$, or coupled to the up-right microscope for the characterization of single fiber laser emission.

\section{Keywords:}

fluorescence $\cdot$ lasers $\cdot$ nanofibers $\cdot$ photonics $\cdot$ waveguides

[1] Y. Xia, P. Yang, Y. Sun, Y. Wu, B. Mayers, B. Gates, Y. Yin, F. Kim, H. Yan, Adv. Mater. 2003, 15, 353.

[2] a) G. A. O'Brien, A. J. Quinn, D. Tanner, G. Redmond, Adv. Mater. 2006, 18, 2379; b) D. O’Carroll, D. lacopino, A. O'Riordan, P. Lovera, É. O’Connor, G. A. O’Brien, G. Redmond, Adv. Mater. 2008, $20,42$.

[3] a) A. M. Morales, C. M. Lieber, Science 1998, 279, 208; b) M. H. Huang, S. Mao, H. Feick, H. Q. Yan, Y. Y. Wu, H. Kind, E. Weber, R. Russo, P. D. Yang, Science 2001, 292, 1897; c) X. Duan, Y. Huang, R. Agarwal, C. M. Lieber, Nature 2003, 421, 241; d) Z. Zhong, D. Wang, Y. Cui, M. W. Bockrath, C. M. Lieber, Science 2003, 302, 1377; e) A. Javey, S.-W. Nam, R. S. Friedman, H. Yan, C. M. Lieber, Nano Lett. 2007, 7, 773.

[4] a) M. Kazes, D. Y. Lewis, Y. Ebenstein, T. Mokari, U. Banin, Adv. Mater. 2002, 14, 317; b) H. Htoon, J. A. Hollingworth, A. V. Malko, R. Dickerson, V. I. Klimov, Appl. Phys. Lett. 2003, 82, 4776.

[5] a) R. Agarwal, K. Ladavac, Y. Roichman, G. Yu, C. M. Lieber, D. G. Grier, Opt. Express 2005, 13, 8906; b) P. J. Pauzauskie, A. Radenovic, E. Trepagnier, H. Shroff, P. Yang, J. Liphardt, Nat. Mater. 2006, 5, 97. 
[6] a) S. Noda, K. Tomoda, N. Yamamoto, A. Chutinan, Science 2000, 289, 604; b) W. L. Barnes, A. Dereux, T. W. Ebbesen, Nature 2003, 424, 824.

[7] D. Li, Y. Xia, Adv. Mater. 2004, 16, 1151.

[8] a) A. Noy, A. E. Miller, J. E. Klare, B. L. Weeks, B. W. Woods, J. J. DeYoreo, Nano Lett. 2002, 2, 109; b) F. Quochi, F. Cordella, R. Orrù, J. E. Communal, P. Verzeroli, A. Mura, G. Bongiovanni, A. Andreev, H. Sitter, N. S. Sariciftci, Appl. Phys. Lett. 2004, 84, 4454; c) D. Pisignano, G. Maruccio, E. Mele, L. Persano, F. Di Benedetto, R. Cingolani, Appl. Phys. Lett. 2005, 87, 123109; d) K. J. Lee, J. H. Ho, Y. Kim, J. Jang, Adv. Mater. 2006, 18, 2216; e) K. Takazawa, Y. Kitahama, Y. Kimura, G. Kido, Nano Lett. 2005, 5, 1293.

[9] J. M. Moran-Mirabal, J. D. Slinker, J. A. DeFranco, S. S. Verbridge, R. Ilic, S. Flores-Torres, H. Abruña, G. G. Malliaras, H. G. Craighead, Nano Lett. 2007, 7, 458.

[10] H. Liu, J. B. Edel, L. M. Bellan, H. G. Craighead, Small 2006, 2, 495.

[11] a) S. Madhugiri, A. Dalton, J. Gutierrez, J. P. Ferraris, K. J. Balkus, Jr., J. Am. Chem. Soc. 2003, 125, 14531; b) D. Li, A. Babel, S. A. Jenekhe, Y. Xia, Adv. Mater. 2004, 16, 2062; c) A. Camposeo, F. Di Benedetto, R. Stabile, R. Cingolani, D. Pisignano, Appl. Phys. Lett. 2007, 90, 143115; d) F. Di Benedetto, A. Camposeo, S. Pagliara, E. Mele, L. Persano, R. Stabile, R. Cingolani, D. Pisignano, Nat. Nanotechnol. 2008, 3, 614.

[12] G. Kwak, S. Fukao, M. Fujiki, T. Sakaguchi, T. Masuda, Chem. Mater. 2006, 18, 5537.

[13] a) D. Li, Y. Wang, Y. Xia, Nano Lett. 2003, 3, 1167; b) D. Li, G. Ouyang, J. T. McCann, Y. Xia, Nano Lett. 2005, 5, 913.

[14] a) E. Zussman, A. Theron, A. L. Yarin, Appl. Phys. Lett. 2003, 82, 973; b) J. Kameoka, R. Orth, Y. N. Yang, D. Czaplewski, R. Mathers, G. W. Coates, H. G. Craighead, Nanotechnology 2003, 14, 1124.

[15] D. Psaltis, Science 2002, 298, 1359.

[16] a) M. L. Chabinyc, D. T. Chiu, J. C. McDonald, A. D. Stroock, J. F. Christian, A. M. Karger, G. M. Whitesides, Anal. Chem. 2001, 73, 4491; b) B. Yao, G. Luo, L. Wang, Y. Gao, G. Lei, K. Ren, L. Chen, Y. Wang, Y. Hub, Y. Qiubc, Lab Chip 2005, 5, 1041.

[17] a) D. O'Carroll, I. Lieberwirth, G. Redmond, Nat. Nanotechnol. 2007, 2, 180; b) F. Quochi, F. Cordella, A. Mura, G. Bongiovanni, F. Balzer, H.-G. Rubahn, Appl. Phys. Lett. 2006, 88, 041106; c) Y. S. Zhao, A. Peng, H. Fu, Y. Ma, J. Yao, Adv. Mater. 2008, 20, 1661.

[18] S. V. Fridrikh, J. H. Yu, M. P. Brenner, G. C. Rutledge, Phys. Rev. Lett. 2003, 90, 144502.
[19] G. Qian, Y. Yang, Z. Wang, C. Yang, Z. Yang, M. Wang, Chem. Phys. Lett. 2003, 368, 555.

[20] A. Bianco, C. Bertarelli, S. Frisk, J. F. Rabolt, M. C. Gallazzi, G. Zerbi, Synth. Met. 2007, 157, 276.

[21] M. Kakade, S. Givens, K. Gardner, K. H. Lee, D. B. Chase, J. F. Rabolt, J. Am. Chem. Soc. 2007, 129, 2777.

[22] M. Majoube, M. Henry, Spectrochim. Acta 1991, 47A, 1459.

[23] G. Wegmann, H. Giessen, A. Greiner, R. F. Mahrt, Phys. Rev. B: Condens. Matter 1998, 57, R4218.

[24] Guided PL emission at the fiber tip is red-shifted compared to light emitted directly from the excited region due to self absorption of the emitted light, which is more relevant for higher energy emitted photons.

[25] D. Carroll, I. Lieberwirth, G. Redmond, Small 2007, 3, 1178.

[26] a) V. G. Kozlov, V. Bulović, P. E. Burrows, S. R. Forrest, Nature 1997, 389, 362; b) R. Gupta, M. Stevenson, A. Dogariu, M. D. McGehee, J. Y. Park, V. Sdranov, A. J. Hegeer, H. Wang, Appl. Phys. Lett. 1998, 73, 3492; c) A. Camposeo, E. Mele, L. Persano, D. Pisignano, R. Cingolani, Opt. Lett. 2006, 31, 1429.

[27] Fibers with diameter below $300 \mathrm{~nm}$ show a weaker coupling of the emitted light into guided modes. This can easily rationalized since for a cylindrical waveguide of core diameter $d_{f}$ the fractional coupled power, $\eta$, is given by $\eta=1-V^{-3}\left[2.405 \exp \left(-V^{-1}\right)\right]^{2}$, where $V=0.5 \mathrm{kd}_{\mathrm{f}}\left(\mathrm{n}^{2}-1\right)^{1 / 2}$ and $\mathrm{k}=2 \pi / \lambda$. See: A. W. Snyder, D. Love, Optical Waveguide Theory, Kluver, Boston 1983. For R6G fibers $(\lambda>500 \mathrm{~nm})$ we estimate that more than $90 \%$ of the emitted light is coupled into the waveguide mode for $d_{\mathrm{f}}>500 \mathrm{~nm}$, whereas $\eta=20 \%$ for $d_{f}=150 \mathrm{~nm}$.

[28] A. K. Sheridan, G. A. Turnbull, A. N. Safonov, I. D. W. Samuel, Phys. Rev. B: Condens. Matter 2000, 62, R11929.

[29] a) P. Del Carro, A. Camposeo, R. Stabile, E. Mele, L. Persano, R. Cingolani, D. Pisignano, Appl. Phys. Lett. 2006, 89, 201105; b) V. Reboud, P. Lovera, N. Kehagias, M. Zelsmann, C. Schuster, F. Reuther, G. Gruetzner, G. Redmond, C. M. Sotomayor Torres, Appl. Phys. Lett. 2007, 91, 151101.

Received: August 8, 2008 Revised: September 30, 2008 Published online: February 2, 2009 